\title{
AWARENESS OF BLADDER FILLING WITH DIVIDED SENSORY TRACT
}

\author{
BY \\ P. W. NATHAN
}

From the Neurological Research Unit of the Medical Research Council, the National Hospital, Queen Square, London

It takes a baby at least two years to learn to interpret certain sensations as indicating that the bladder needs to be emptied. When the afferent pathway from the bladder in the spino-thalamic tract has been divided, these specific sensations are no longer experienced. However, when this pathway from the bladder has been divided and the normal sensation underlying the desire to micturate has thereby been removed, it is surprising to find that many patients experience other sensations related to bladder filling and emptying; and they can learn to interpret such sensations, which intrinsically do not feel as though they are related to the desire to micturate, as an indication that the bladder needs to be emptied. The purpose of this paper is to describe and draw attention to the more common of these sensations, to report an investigation of its mechanism, and to present evidence on the pathway by which the impulses subserving it reach the central nervous system.

In patients with total division of the spinal cord the occurrence of a poorly localized abdominal sensation indicating a full bladder has been reported by Bálint and Benedict (1906), Riddoch (1921), Denny-Brown and Robertson (1933), Voris and Landes (1940), Donovan (1947), and Kuhn (1950); it has been reported in total lesions of the cauda equina by Kocher (1896) and Denny-Brown and Robertson (1933); and in patients with division of the lateral part of the spino-thalamic tract Nathan and Smith (1951) recorded the presence of this and other substitute sensations. The cases reported by Kuhn are of particular interest, as he made some observations on the nature of the stimulus that may give rise to the sensation in patients with proved total division of the cord ; reference to his material will be made throughout this study.

The abdominal sensation is not the only sensation that may act as a substitute for the normal sensation underlying the need to micturate. Occasionally, after the spino-thalamic tract has been divided, there are spontaneous tingling paraesthesiae in the lower part of the body; these may be accentuated when the bladder is full. In some patients there may be reference of sensation from below the level of the surgical lesion to the parts of the body above it; such referred sensations may originate in the bladder. As was previously mentioned by Nathan and Smith (1951), a very full bladder may cause one of the following sensations : a frontal headache, a feeling of being about to shiver or of actual shivering movements, a feeling of a need to take a deep breath; these are the sensations that accompany the reflex effects described in total cord division by Guttmann and Whitteridge (1947). There are also the sensations arising in the urethra that occur immediately before micturition; these were also briefly described by Nathan and Smith.

Unless the clinician is aware of the possible existence of these substitute sensations, he may be misled by the answer to the question whether the patient knows when he needs to micturate or not. For patients having these substitute sensations may not explain spontaneously that although they know when they need to empty their bladders they have not acquired this knowledge in the usual way; it may be necessary to tell them of the existence of the normal sensation of needing to micturate and of this vague abdominal sensation before they will state that this is the sensation which they experience. Further, these sensations are useful, as they can form adequate substitutes for the normal sensation when this is absent.

This vague abdominal sensation was present in 11 of 20 patients who had the operation of bilateral antero-lateral cordotomy ; it was also experienced by one patient with a thrombosis of the anterior spinal artery, causing extensive destruction of the afferent pathway from the bladder.

\section{The Nature of the Sensation}

The most common of the substitute sensations related to bladder filling is a vague feeling in the abdomen, poorly localized and difficult to define. All patients say that it is a sensation that they have not previously experienced. Many of them, when 
they first experience it, believe that they are distended with wind. One young man described it as follows: "When my belly feels very full, as though I have eaten a lot, then I know I have to empty my bladder." Sometimes the sensation is described as an ache, which may have an unpleasant component, but it is uncomfortable rather than painful. All patients agree that this feeling of abdominal fullness does not seem to be connected with the bladder. It is experience that teaches them to use this non-specific sensation as an indicator of bladder fullness. The sensation is not usually noticed immediately after division of the spino-thalamic tracts ; it may take days or weeks before the patient becomes aware of it.

\section{The Origin of the Sensation}

As this sensation comes on when the bladder is full and goes after the bladder has been emptied, it is reasonable to look for its source in the bladder. However, certain observations were made that showed that the bladder could not be the only source of the sensation. Accordingly the following four possible sources of the nervous inpulses giving rise to the sensation were examined : the bladder; an increase in intra-abdominal pressure ; the pelvic floor; and the rectum.

Cases will now be presented as illustrations of the evidence concerning these four possible sources of the sensation.

The Bladder.- - It seems to be that any inflow into the central nervous system of impulses from the bladder may give rise to this sensation. Undoubtedly the impulses consequent on stretching and contracting of the bladder muscle fibres form one main source of the sensation.

An increase in bladder pressure, as occurs during the performance of cystometrography and cystoscopy, has on innumerable occasions given rise to the sensation. This sensation, however, is not so closely related to changes in bladder pressure as is that occurring in the healthy subject interpreted as the need to micturate. It may not become more intense with sudden increases of bladder pressure and may take several minutes to disappear after the bladder has been emptied.

The nature of the sensation and its rather vague relation to the changing bladder pressure is well illustrated by the following case.

Case 10*.-This young man had an extensive sarcoma of the left innominate bone; for the pain from this condition, a bilateral cordotomy was performed. A cystometrogram carried out before the operation

* The numbers of the cases are the same throughout this and all other papers by Nathan and Smith.
Figs. 1a and $1 \mathrm{~b}$.-Pre- and post-operative cystometrograms.
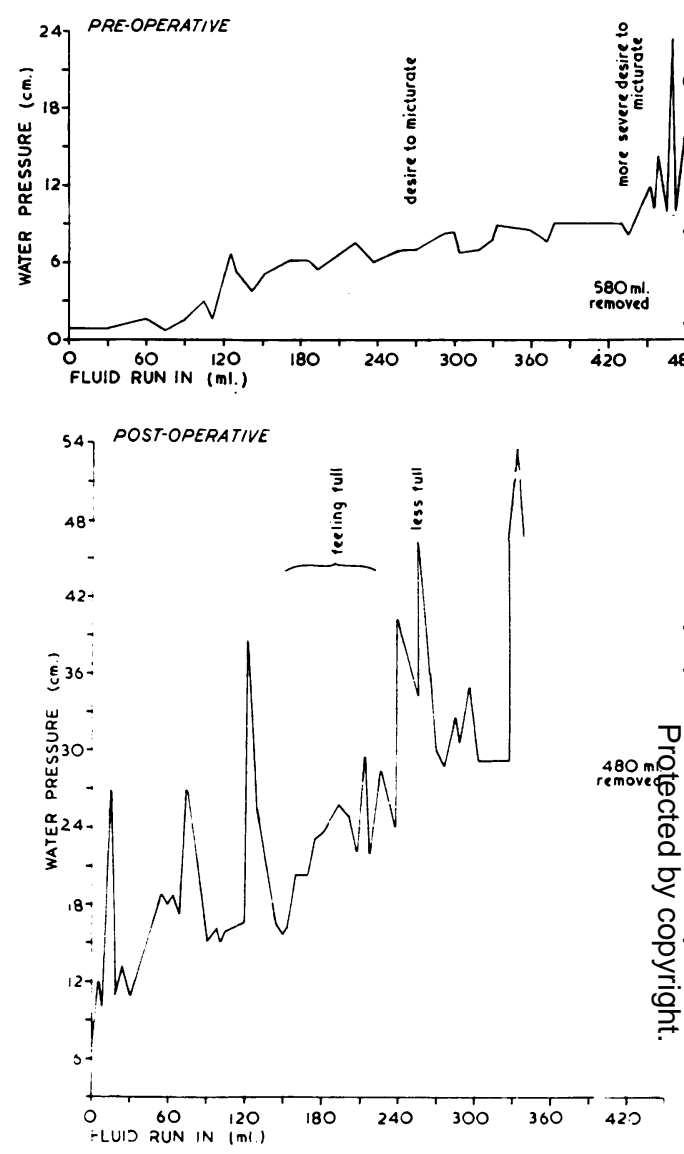

(Fig. 1a) was normal; the bladder had a large capacis but one within the range of the normal.

Following operation there was analgesia throughou the right side of the body below the eleventh thoracic and throughout the left side below the tenth thoracte dermatome. The patient had retention of urine, ne desire to micturate, and no pain when the bladder was. allowed to become very distended immediately after the operation. He soon found, however, that he could tett when his bladder was full as he got a sensation of fullness. This was vague and seemed to be generally within the abdomen; it was the same as the feeling of being distended with wind ; it was by experience that he learn that it indicated that his bladder needed emptying.

A cystometrogram was carried out six weeks after the operation (Fig. 1b). The shape of the curve does not. concern us here. No sensation of a normal kind occurred either with the gradual filling of the bladder or with the large spontaneous contractions. The words "feeling full " refer to the vague sensation of abdominal fullness it was experienced when $200 \mathrm{ml}$. of fluid had been rug into the bladder ; such an amount of fluid could not have 
over-distended the bladder. It will also be seen from this figure that the sensation diminished as the filling and the pressure of the bladder increased.

From this typical case it can be concluded that slowly filling the bladder in a normal manner induces the abdominal sensation. It is also seen that the sensation is not proportional to the amount of fluid in the bladder or to the amount of reflex contraction of the bladder. These points are shown more clearly in the next case.

Case 9.-This patient developed a thrombosis of the anterior spinal artery during the second stage of an abdominal sympathectomy. Analgesia resulted throughout the body below the second lumbar dermatome. The bladder had minimal tone, manifested no spontaneous contractions and failed to respond to stretch by an increase in pressure.

When the bladder was washed out, although the pressure was not allowed to rise above $7 \mathrm{~cm}$. of water, the patient experienced the sensation of abdominal distension ; and the sensation, once aroused in this way, would always continue for many minutes after the lavage had ceased and the bladder had been emptied.

To observe how the character of the vague sensation might change as the bladder became over-distended, the patient was left for 12 hours without being catheterized. She had no distress, no pain, and no normal sensation of needing to micturate. She got " a vague feeling of fullness, a discomfort, not a pain" situated somewhere in the abdomen between the umbilicus and the pubis; this sensation did not become more intense or painful with the increasing distension.

From many observations made during cystometrography and cystoscopy in the 12 patients, it has been concluded that this abdominal sensation may arise from normal physiological stretching of the bladder. This conclusion was from the outset to have been expected, although the sensation does not feel to the patient as though it is connected with the bladder.

However, it may be surprising to realize that this same abdominal sensation may be experienced after the bladder has been removed. The presence of this substitute sensation in a patient without a bladder is illustrated by the following case.

Case 60.-A man with a carcinoma of the bladder was operated upon by Mr. David Wallace, who performed the following ingenious operation. He carried out a total cystectomy, dividing the urethra through its prostatic part; he then mobilized a piece of ileum, transplanted the ureters into it, and joined the loop of ileum to the divided urethra. Thus the patient was provided with a new bladder.

Two to three weeks after the operation, the patient found that he knew when his artificial bladder was becoming full, as he experienced " a blown-up feeling" in the lower abdomen. This sensation was not painful; it was not the sensation of colic of the bowel ; and it was not the sensation of wind, "because wind hurts".

Thus this patient experienced a substitute abdominal sensation which he came to interpret as indicative of bladder fullness, although the actual bladder had been completely removed. It is to be emphasized that, as far as one can judge from verbal descriptions by patients, this sensation originating elsewhere than the bladder was the same as that originating in the bladder.

Although it has been concluded that the sensation may originate in the bladder, it may also originate from sources other than the bladder.

An Increase in Intra-abdominal Pressure.-There is no doubt that the sensation may be aroused by an increase of the intra-abdominal pressure; and there is also no doubt that it does not invariably so arise. The role of an increase in the intra-abdominal pressure in increasing the sensation is illustrated by the following case.

Case 19.- This man had a bilateral cordotomy for the pain caused by a carcinoma of the bronchus. Following the operation there was complete analgesia throughout the body below the fourth thoracic dermatome.

Ten weeks after the operation, the bladder was filled through a catheter. When spontaneous contractions occurred, the patient had a substitute sensation in the form of pricking in the neighbourhood of the pubis and in the lower abdomen; this sensation would continue for minutes after the pressure was reduced by lowering the reservoir of fluid. When the patient had this sensation, it could be increased by gentle pressure on the upper abdomen. As this pressure was applied when the bladder was actively contracting, no increase in the intra-vesical pressure occurred, as shown by manometric readings taken during this procedure.

However, it is also clear that the sensation may arise without there being an increase in intraabdominal pressure. On many occasions patients who were subject to this sensation were induced to strain as though defaecating or as though trying to expel urine ; such a manoeuvre increased the intraabdominal pressure, but did not induce this abdominal sensation.

It is also clear that when the sensation arises in the bladder, this is not due to a concomitant rise in intra-abdominal pressure. For instance, the sensation sometimes occurred when only 200 to $300 \mathrm{ml}$. of urine was in the bladder and when the bladder was contracting firmly; such small amounts of fluid in the bladder could not cause a rise in the intra-abdominal pressure.

Further evidence that the sensation may arise without any increase in the intra-abdominal pressure is provided by the cases of total transection of the spinal cord, published by Kuhn. In four patients 
(D.La., J.D., J.B., and McC.), Kuhn obtained the feeling of fullness in the lower abdomen or a tightening in this region or aching below the umbilicus when he stretched the anal sphincter. As this stretching is not associated with an increase of the intra-abdominal pressure, it follows that the abdominal sensation does not necessarily arise from an increase in the intra-abdominal pressure.

The Pelvic Floor.-That the sensation did not originate from the pelvic floor or the perineal musculature was made clear by the following case.

Case 61.-Mrs. R., before admission, had had an intrathecal injection of alcohol in order to alleviate the pain from a carcinoma of the cervix uteri. This had destroyed all the anterior and posterior roots of the cauda equina. The perineal and pelvic musculature had no tone and could not be contracted voluntarily. The patient was not incontinent of urine ; she could empty the bladder by pressing with the hands on the lower abdomen. She had the substitute sensation so clearly that she always made use of it to empty the bladder.

From this case, it is concluded that the sensation does not originate in the contraction of the muscles of the pelvic floor.

The Rectum.-There is no evidence that the sensation can originate in the rectum. No patient experiencing the sensation has lost it after a full rectum was emptied. In many patients the sensation disappeared on emptying the bladder, though the rectum remained full of hard masses of faecal material.

To investigate further the rectum as a possible source of the sensation, a balloon was passed into the rectum of several patients and then distended. Though the patients usually became aware of a sensation of distension of the rectum, it was quite unlike the vague abdominal sensation under consideration. Finally, four patients who previously had the rectum excised experienced the substitute sensation; in them, the sensation could not have arisen in the rectum.

Thus an enquiry into the origin of this abdominal sensation shows that it can originate in the bladder, and/or from an increase in intra-abdominal pressure while the bladder pressure remains constant. There is no evidence that it originates in the floor of the pelvis or the rectum.

\section{The Pathway of Impulses Subserving the Sensation}

It has been shown by Nathan and Smith (1951) that after the afferent pathway from the bladder in the spino-thalamic tract has been divided this abdominal sensation may be experienced. The possible remaining pathways which may subserve this substitute sensation will now be considered, first when it arises from the bladder, and seconकly when it arises from an increase in intra-abdomigal pressure.

The Pathway from the Bladder.-Two possif̣le routes must be considered : first, a pathway within the spinal cord, and secondly, one outside the cond. The route outside the cord would be afferent nerges running in company with the sympathetic ner丞es and chains; these nerves would eventually reah the spinal cord via the posterior roots in the upser thoracic region.

Observations reported by Kuhn (1950) and $\frac{E_{\text {Wy }}}{y}$ Beck (1949) on patients with total transections $\rightrightarrows f$ the cord make it clear that there are afferent fibes subserving sensation within the sympathetic chagin. In Kuhn's patients with division of the cord, the only possible afferent pathway from the pelvic structures to the upper spinal cord was that running with the sympathetic nerves and chain. In Fis patients, the impulses giving rise to the sensation under discussion must have made use of this pathway. Further evidence for conduction via a pathuray incorporated in the sympathetic chain comes forth the well studied case reported by Beck. In thisacase the division of the cord had been confirmes at operation: "Immediately below the third thoraeic segment there is division of the cord. There is a defect of $2 \mathrm{~cm}$. between both ends of the cord ". Oार्मुe bullet that had divided the cord had also dikded the sympathetic chain on the right and had destrồंध口 the right fourth thoracic sympathetic ganglion. FRs patient, "shortly before micturition", whigh occurred reflexly when 200 to $300 \mathrm{ml}$. of urine wh in the bladder, experienced " a feeling of cramp the neighbourhood of the bladder, which alws slowly ascended along the left side of the trunk. $\bar{g} t$ went away after micturition". The fact that the sensation was felt mounting up the left side and that the right sympathetic chain had been divided may suggest that the pathway being used in this case wa्as one running along the left sympathetic chain.

It thus appears that there are afferent nerves running with the sympathetic system which conduet impulses subserving sensation from pelvic structures, and that the sensory function of these nerves becomes manifest when the spinal cord has been totally divided at a level caudal to the entry of the fibres accompanying the sympathetic chain. Further, as there was a proved total division of the cot immediately below the third thoracic segment Beck's case and at the fourth thoracic segment of Kuhn's case McC., it seems that these afferens nerves must reach the spinal cord at a level crania to the fourth thoracic segment. Thus in thos patients with a total division of the cord, the afferent 
pathway for the impulses subserving this abdominal sensation must be via the fibres accompanying the sympathetic system. In the patients investigated here, those in whom the spino-thalamic tracts had been divided, it is probable that the same sensation was subserved by the same pathway ; it is possible, however, that in these patients the pathway was via the sacral posterior roots and the posterior columns of the cord.

The Pathway when the Sensation Arises from an Increase in Intra-abdominal Pressure.-When the sensation arises from an increase in the intraabdominal pressure, the relevant impulses may travel via afferent nerves accompanying the sympathetic system, or, in some cases, they may travel via the posterior roots and then the posterior columns. There are, however, two other possible pathways to consider : the branches of the phrenic nerve supplying the abdominal surface of the diaphragm and the peritoneum covering it, and the coeliac branches of the vagus. It would seem to be unlikely that the phrenic nerve is used as a pathway, for no case was seen in which a sensation was felt in the shoulder-tip; if the phrenic nerve-endings were being stimulated, it is likely that the sensation would be referred to this territory.

It has not been possible to obtain evidence as to which pathway is used; for in those patients in whom the sensation could be shown to occur in association with a rise in intra-abdominal pressure, the branches of the vagus, many sympathetic fibres, and the posterior columns were intact.

\section{Summary}

A sensation is described which occurs after the normal afferent pathways from the bladder have been divided. Patients may learn to use this sensation as a substitute for the normal sensation giving rise to the desire to micturate.

The possible sources of this sensation have been investigated. It is concluded that the impulses subserving it may originate in the bladder and/or from an increase in intra-abdominal pressure.

When the cord is totally divided, the impulses subserving this sensation are conducted via afferent nerves accompanying the sympathetic nerves; it is shown that some of these nerves enter the spinal cord at a level cranial to the fourth thoracic segment. When only the spino-thalamic tracts are divided, the impulses may follow the same path, or they may run in the posterior columns. When the sensation originates from an increase in intra-abdominal pressure, it is possible that the coeliac branches of the vagus also conduct the relevant impulses.

I wish to thank Dr. E. A. Carmichael for his helpful interest, Mr. Wylie McKissock for his continuous enthusiastic cooperation, and Mr. O'Connell, Mr. David Wallace, and Dr. Alan Barham-Carter, who were so kind as to allow me to investigate their cases.

\section{REFERENCES}

Bálint, R., and Benedict, H. (1906). Dtsch. Z. Nervenheilk., 30, 1. Beck, K. (1949). Ibid., 160, 55.

Denny-Brown, D., and Robertson, E. G. (1933). Brain, 56, 149

Donovan, H. (1947). Lancet, 1, 515.

Guttmann, L., and Whitteridge, D. (1947). Brain, 70, 361.

Kocher, T. (1896). Mitt. Grenzgeb. Med. Chir., 1, 415.

Kuhn, R. A. (1950). Brain, 73, 1.

Nathan, P. W., and Smith, M. C. (1951). Journal of Neurology, Neurosurgery and Psychiatry, 14, 262.

Riddoch, R. (1921). J. Physiol., Lond., 54, 134

Voris, H. C., and Landes, H. E. (1940). Arch. Neurol. Psychiat., (Chicago), 44, 118 . 